\title{
Burst-by-Burst Adaptive Turbo-Coded Radial Basis Function-Assisted Decision Feedback Equalization
}

\author{
M. S. Yee, Associate Member, IEEE, T. H. Liew, and Lajos Hanzo, Senior Member, IEEE
}

\begin{abstract}
The performance of the proposed radial basis function (RBF) assisted turbo-coded adaptive modulation scheme is characterized in a wideband channel scenario. We commence by introducing the novel concept of the Jacobian RBF equalizer, which is a reduced-complexity version of the conventional $R B F$ equalizer. Specifically, the Jacobian logarithmic RBF equalizer generates its output in the logarithmic domain and hence it can be used to provide soft outputs for the turbo-channel decoder. We propose using the average magnitude of the log-likelihood ratio (LLR) of the bits in the received transmission burst before channel decoding as the channel quality measure for controlling the mode-switching regime of our adaptive scheme.
\end{abstract}

Index Terms-Adaptive modulation, AQAM, decision feedback equalizer, DFE, Jacobian logarithm, neural network, radial basis function, RBF, turbo coding.

\section{BACKGROUND}

A DAPTIVE quadrature amplitude modulation (AQAM) was originally proposed by Webb and Steele [1] and it was further developed by Sampei et al. [2], Goldsmith et al. [3], and Torrance et al. [4] in the context of nondispersive channels. Wong et al. [5] extended these contributions to dispersive wideband channels with the aid of a burst-by-burst (BbB)adaptive Kalman filtered AQAM scheme. Radial basis function (RBF)-assisted decision feedback equalization (DFE) has been documented in the context of fixed-mode modulation schemes [6], [7]. However, there is little information concerning its potential in either $\mathrm{BbB} \mathrm{AQAM}$ or in forward error correction (FEC)-coded scenarios, despite the advantageous interactions of RBF-aided DFE BbB AQAM in conjunction with turbo FEC, which were demonstrated in our preliminary work [8]. Hence in this contribution, we set out to document these interactions.

The symbol-spaced channel output can be defined by

$$
v_{k}=\sum_{n=0}^{L} f_{n} I_{k-n}+\eta_{k}=\tilde{v}_{k}+\eta_{k}, \quad-\infty \leq k \leq \infty
$$

where

$\left\{f_{n}\right\} \quad n=0,1, \ldots, L ;$ channel impulse response (CIR);

$\left\{I_{k}\right\} \quad$ channel's input sequence;

$\left\{\tilde{v}_{k}\right\} \quad$ noiseless channel output.

Paper approved by C. Schlegel, the Editor for Coding Theory and Techniques of the IEEE Communications Society. Manuscript received April 5, 2000; revised March 20, 2001. This work was supported by the EPSRC, the U.K., and the Commission of the European Communities, Brussels, Belgium.

The authors are with the Department of Electronics and Computer Science, University of Southampton, Highfield Southampton SO17 1BJ, U.K. (e-mail: lh@ecs.soton.ac.uk).

Publisher Item Identifier S 0090-6778(01)10163-7.
The channel output observed by the linear $m$ th-order equalizer can be written in vectorial form as $\mathbf{v}_{k}=\left[\begin{array}{lll}v_{k} & v_{k-1} & \cdots\end{array}\right.$ $\left.v_{k-m+1}\right]^{T}$, and hence we can say that the equalizer has an $m$-dimensional channel output observation space. For a CIR of length $L+1$ and $\mathcal{M}$ number of QAM constellation points, there are hence $n_{s}=\mathcal{M}^{L+m}$ possible noiseless channel output vector combinations $\tilde{\mathbf{v}}_{k}=\left[\begin{array}{lllll}\tilde{v}_{k} & \tilde{v}_{k-1} & \cdots & \tilde{v}_{k-m+1}\end{array}\right]^{T}$ due to the channel input sequence $\mathbf{I}_{k}=\left[\begin{array}{lllll}I_{k} & I_{k-1} & \cdots & I_{k-m-L+1}\end{array}\right]^{T}$. The possible noiseless channel output values or particular points in the observation space will be referred to as the channel states, $\mathbf{r}_{i}, 1 \leq i \leq n_{s}$. Gibson, Siu, and Cowan [9] interpreted the equalization problem as a geometric classification process. They demonstrated how a neural network, as a nonlinear classifier, classifies the channel state pattern in the observation space according to the symbol that deemed to have been transmitted. Since then, various nonlinear structures applied to the problem of adaptive channel equalization have been investigated, such as the multilayer-perceptron network [9]-[11], polynomial-perceptron structure [12], the functional link structure [13], the self-organizing map [14], the recurrent network of [15] and RBF networks [16]. Chen, Mulgrew, McLaughlin, and Grant [6], [16], [17] promoted the employment of RBF based equalizers, which exhibit a structure identical to that of the optimal Bayesian symbol-decision based equalizer. Therefore, RBF equalizers can rely on optimal detection theory [18]. In this contribution, we use a decision feedback assisted RBF equalizer, which involves its previous decisions for either RBF subset center selection [17] or space translation [19], [20], in order to reduce its computational complexity. Both versions of the DFE realize the same optimal solution, but the space translation based version [19], [20] requires less storage for the RBF centers, since the centers corresponding to different decision feedback vectors are equivalent in the translated space [19], [20]. Therefore the latter version is preferred in hardware implementations.

BbB AQAM schemes [21] employ a higher-order modulation scheme in a certain transmission burst, when the channel quality is favorable, in order to increase the throughput and conversely, a more robust, lower-order modulation scheme is utilized in those transmission bursts, where the instantaneous channel quality drops [1]. The performance benefits of the RBF DFE [17] have been documented in the context of AQAM over dispersive wideband mobile channels in [22], [23], demonstrating that a certain target bit error rate (BER) can be maintained across a wide range of channel signal-to-noise ratios (SNRs) by adjusting the modulation mode between 1 and $6 \mathrm{bit} / \mathrm{symbol}$ (BPS) according to the instantaneous channel quality. As the SNR improved, an increased BPS throughput 
was achieved. Furthermore, since the number of BPS was adjusted on a BbB basis, in order to prevent inflicting bursts of errors due to using high-throughput but vulnerable AQAM modes under low instantaneous SNR conditions, the demodulated bit stream becomes more amenable to channel decoding. Hence the main motivation of this contribution is to study the interactions of RBF-assisted BbB AQAM with FEC codingin particular, with the most powerful family of known FEC codes, namely turbo codes [24].

The outline of the paper is as follows. Section II introduces the concept of Jacobian logarithmic RBF equalizers, while Section III provides a system overview. Sections IV and V present our performance studies of fixed and BbB AQAM schemes, respectively, before concluding in Section VI.

\section{JACOBIAN LOGARITHMIC RBF EQUALIZER}

In this section, before we discuss the proposed joint RBF DFE $\mathrm{BbB}$ AQAM and turbo-coding system, we will introduce the Jacobian logarithmic RBF DFE, which generates the output of the RBF network in logarithmic form in order to assist the Log MAP algorithm [25] used in our turbo codec and to reduce its computational complexity.

The overall response of the $m$-input RBF network having $M$-hidden nodes in conjunction with the Gaussian activation function is as follows [26]:

$$
f_{R B F}\left(\mathbf{v}_{k}\right)=\sum_{i=1}^{M} w_{i} \exp \left(-\left|\mathbf{v}_{k}-\mathbf{c}_{i}\right|^{2} / \rho\right)
$$

where $\rho, w_{i}$ and the $m$-component vector $\mathbf{c}_{i}, i=1, \ldots, M$ are the width, weights, and centers of the RBF, respectively, [26]. The equalizer's input is the $m$-dimensional channel output vector at signaling instant $k$. The RBF network has a similar structure to the optimal Bayesian equalizer [16] and thus it was proposed for equalization in [6], [16], [17]. The output of the RBF equalizer based on the optimal Bayesian decision function-where the RBF centers are assigned the values of the channel states $\mathrm{r}_{i}$, the RBF weights correspond to the a priori probability of the channel states $p_{i}$ and the RBF width is given by $2 \sigma_{\eta}^{2}$, with $\sigma_{\eta}^{2}$ being the channel noise variance-provides the conditional density function of each legitimate $\mathcal{M}$-QAM symbol [16]. The a posteriori probability of each symbol can be evaluated from their conditional probability density function (pdf) and the highest probability symbol becomes the detected symbol.

The Bayesian-based RBF equalizer [16] has a high computational complexity due to the nonlinear exponential functions in (2) and due to the high number of additions/subtractions and multiplications/divisions required for the estimation of each symbol.

In this section-based on the approach often used in turbo codes-we propose generating the output of the RBF network in logarithmic form by invoking the so-called Jacobian logarithm [27], [28], in order to avoid the computation of exponentials and to reduce the number of multiplications performed. We will refer to the RBF equalizer using the Jacobian logarithm as the Jacobian logarithmic RBF equalizer. Below we will present this idea in more detail. The Jacobian logarithmic relationship for exponential summation is given by [27]:

$$
\ln \left(\sum_{k=1}^{n} e^{\lambda_{k}}\right)=J\left(\lambda_{n}, J\left(\lambda_{n-1}, \ldots J\left(\lambda_{3}, J\left(\lambda_{2}, \lambda_{1}\right)\right) \cdots\right)\right)
$$

where the Jacobian logarithm function $J\left(\lambda_{1}, \lambda_{2}\right)$ is given by

$$
\begin{aligned}
J\left(\lambda_{1}, \lambda_{2}\right) & =\ln \left(e^{\lambda_{1}}+e^{\lambda_{2}}\right) \\
& =\max \left(\lambda_{1}, \lambda_{2}\right)+\ln \left(1+e^{-\left|\lambda_{1}-\lambda_{2}\right|}\right) \\
& \approx \max \left(\lambda_{1}, \lambda_{2}\right)+\varphi_{c}\left(\left|\lambda_{1}-\lambda_{2}\right|\right)
\end{aligned}
$$

The correction function $\varphi_{c}(x)=\ln \left(1+e^{-x}\right)$ can be tabulated in a look-up table, in order to reduce the computational complexity [27]. Expressing (2) in a logarithmic form and substituting in the Jacobian logarithm, we obtain

$$
\begin{aligned}
\ln \left(f_{\mathrm{RBF}}\left(\mathbf{v}_{k}\right)\right) & =\ln \left(\sum_{i=1}^{M} w_{i} \exp \left(-\left|\mathbf{v}_{k}-\mathbf{c}_{i}\right|^{2} / \rho\right)\right) \\
& =\ln \left(\sum_{i=1}^{M} \exp \left(\ln \left(w_{i}\right)\right) \exp \left(-\left|\mathbf{v}_{k}-\mathbf{c}_{i}\right|^{2} / \rho\right)\right) \\
& =\ln \left(\sum_{i=1}^{M} \exp \left(w_{i}^{\prime}+\nu_{i k}\right)\right) \\
& =\ln \left(\sum_{i=1}^{M} \exp \left(\lambda_{i k}\right)\right) \\
& =J\left(\lambda_{M k}, J\left(\lambda_{(M-1) k}, \ldots J\left(\lambda_{2 k}, \lambda_{1 k}\right) \cdots\right)\right)
\end{aligned}
$$

where $w_{i}^{\prime}=\ln \left(w_{i}\right)$, which can be considered as a transformed RBF weight. Furthermore, we used the shorthand $\nu_{i k}=-\mid \mathbf{v}_{k}-$ $\left.\mathbf{c}_{i}\right|^{2} / \rho$ and $\lambda_{i k}=\nu_{i k}+w_{i}^{\prime}$. By introducing the Jacobian logarithm, every weighted summation of two exponential operations in (2) is substituted according to (4) by an addition, the subtraction $\left|\lambda_{1}-\lambda_{2}\right|$, the $\varphi_{c}\left(\left|\lambda_{1}-\lambda_{2}\right|\right)$ table look-up and a $\max \left(\lambda_{1}, \lambda_{2}\right)$ operation, thus reducing the computational complexity. The computational complexity of the logarithmic RBF DFE, employing the Jacobian algorithm, with feedforward order of $m$, feedback order of $n$ and having $n_{s, j}=\mathcal{M}^{m+L-n}$ hidden RBF nodes per equalized output sample, is given in Table I.

Most of the computational load upon evaluating the RBF response in (2) arises from computing the Euclidean norm term $\nu_{i k}=-\left|\mathbf{v}_{k}-\mathbf{c}_{i}\right|^{2}$ in (2), and the associated total complexity will depend on the number $M$ of RBF centers and on the dimension $m$ of both the RBF center vector $\mathbf{c}_{i}$ and the channel output vector $\mathbf{v}_{k}$. Exploiting the fact that the elements of the vector of noiseless channel outputs constituting the channel states $\mathbf{r}_{i}, i=$ $1, \ldots, n_{s}$ correspond to the convolution of a sequence of $(L+$ 1) transmitted symbols and $(L+1)$ CIR taps-where these vector elements will be referred to as the scalar channel states $r_{l}, l=1, \ldots, n_{s, f}\left(=\mathcal{M}^{L+1}\right)$ —we could use Patra's and Mulgrew's method [29] for reducing the computational load arising 
TABLE I

COMPUTATIONAL COMPLEXITY PER EQUALIZED OUTPUT SAMPLE OF AN $\mathcal{M}$-ARY JACOBIAN LOGARITHMIC RBF DFE WITH $m$ INPUTS AND $n_{s, j}$ HIDDEN RBF NODES BASED ON (5)

Determine the feedback state

$n_{s, j}(2 m+2)-2 \mathcal{M}, \quad$ subtraction and addition

$n_{s, j} m \quad$ multiplication

$n_{s, j} \quad$ division

$n_{s, j}-\mathcal{M}+1 \quad \max$

$n_{s, j}-\mathcal{M} \quad$ table look-up

from evaluating the Euclidean norm $\nu_{i k}$ in (5). Expanding the term $\nu_{i k}$ gives

$$
\begin{aligned}
\nu_{i k}=- & \frac{\left|\mathbf{v}_{k}-\mathbf{c}_{i}\right|^{2}}{\rho} \\
=- & {\left[\frac{\left(v_{k}-c_{i 0}\right)^{2}}{\rho}+\cdots+\frac{\left(v_{k-j}-c_{i j}\right)^{2}}{\rho}\right.} \\
& \left.+\cdots+\frac{\left(v_{k-m+1}-c_{i(m-1)}\right)^{2}}{\rho}\right], \\
& \quad i=1, \ldots, M, \quad k=-\infty, \ldots, \infty
\end{aligned}
$$

where $v_{k-j}$ is the delayed received signal and $c_{i j}$ is the $j$ th component of the RBF center vector $\mathbf{c}_{i}$, which takes the values of the scalar channel outputs $r_{l}, l=1, \ldots, n_{s, f}$. Note from (6) that $\nu_{i k}$ is a summation of the delayed components, $\left(\left(v_{k}-\right.\right.$ $\left.\left.c_{i j}\right)^{2}\right) / \rho$ and the scalar centers $c_{i j}$ take the values of the scalar channel outputs $r_{l}, l=1, \ldots, n_{s, f}$. Thus, we could reduce the computational complexity of evaluating (6) by precalculating $d_{l}=\left(\left(v_{k}-r_{l}\right)^{2}\right) / \rho, l=1, \ldots, n_{s, f}$ for all the $n_{s, f}$ possible values of the scalar channel outputs $r_{l}, l=1, \ldots, n_{s, f}$ and storing the values. From (6) the value of $\nu_{i k}$ can be obtained by summing the corresponding delayed values of $d_{l}$, which we will define as

$$
d_{l j}=\frac{\left(v_{k-j}-r_{l}\right)^{2}}{\rho}, \quad l=1, \ldots, n_{s, f}, \quad j=0, \ldots, m-1
$$

yielding

$$
\nu_{i k}=\sum_{\substack{j=0 \\ c_{i j}=r_{l}}}^{m-1} d_{l j}, \quad i=1, \ldots, M, \quad k=-\infty, \ldots, \infty .
$$

The reduced complexity computation of $\nu_{i k}$ in (6) based on the scalar channel outputs can be represented as in Fig. 1. The multiplexer (Mux) maps $d_{l j}$ corresponding to the scalar center $r_{l}$ to the contribution of the vector center's component $c_{i j}$.

The reduced computational complexity per equalized output sample of an $\mathcal{M}$-ary Jacobian DFE with $m$ inputs, $n_{s, j}=$ $\mathcal{M}^{m+L-n}$ hidden RBF nodes derived from $n_{s, f}=\mathcal{M}^{L+1}$ scalar centers is given in Table II. Comparing Tables I and II, we observe a substantial computational complexity reduction, especially for a high feedforward order $m$, since $n_{s, f}<n_{s, j}$, if $m-n>1$. For example, for the 16 QAM BbB AQAM mode

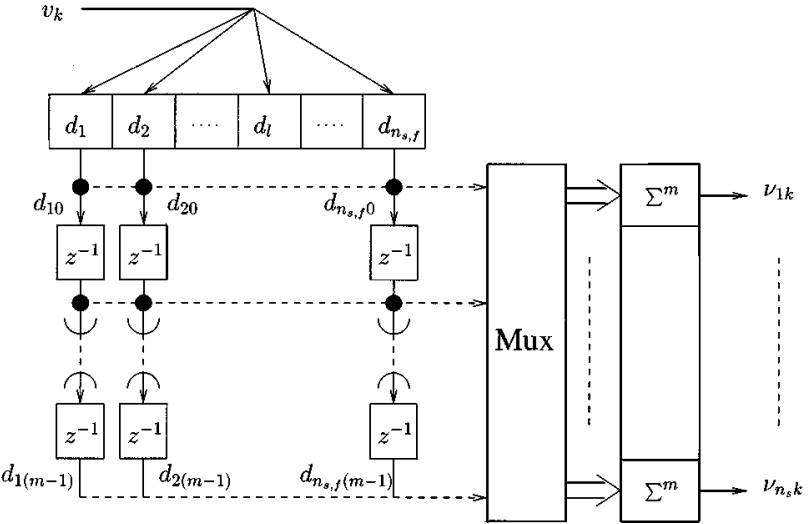

Fig. 1. Reduced-complexity computation of $\nu_{i k}$ in (6) for substitution in (5) based on the scalar channel outputs, $r_{l}, l=1, \ldots, n_{s}, f$.

TABLE II

REDUCED-COMPUTATIONAL COMPLEXITY PER EQUALIZED OUTPUT SAMPLE OF AN $\mathcal{M}$-ARY JACOBIAN LOGARITHMIC RBF DFE BASED ON THE SCALAR CENTERS. THE JACOBIAN RBF DFE BASED ON EQUATION (5) HAS $m$ INPUTS AND $n_{s, j}$ HIDDEN RBF NODES, WHICH ARE DERIVED FROM THE $n_{s, f}$ NUMBER OF SCALAR CENTERS

\begin{tabular}{ll}
\hline \multicolumn{2}{l}{ Determine the feedback state } \\
$n_{s, j}(m+2)-2 \mathcal{M}+n_{s, f}$ & subtraction and addition \\
$n_{s, f}$ & multiplication \\
$n_{s, f}$ & division \\
$n_{s, j}-\mathcal{M}+1$ & max \\
$n_{s, j}-\mathcal{M}$ & table look-up \\
\hline
\end{tabular}

of our system we have $n_{s, f}=256$ and $n_{s, j}=256$ for the RBF DFE equalizer parameters of $m=2, n=1$ and $\tau=1$. The total complexity reduction is by a factor of about 1.3 . If we increase the RBF DFE feedforward order and use the equalizer parameters of $m=3, n=1$ and $\tau=2$-which gives a better BER performance - then we have $n_{s, f}=256$ and $n_{s, j}=4096$ - and the total complexity reduction is by a factor of about 2.1. The computational complexity can be further reduced by neglecting the RBF scalar centers situated far from the received signal $v_{k}$, since the contribution of the RBF scalar centers $r_{l}$ to the decision function of (2) is inversely related to their distance from the received signal $v_{k}$, as observed by Patra [29].

The performance of the RBF DFE and that of its Jacobian approximation was virtually identical, as demonstrated in [8]. However, due to lack of space here no explicit comparisons were included. Having presented the proposed reduced complexity Jacobian logarithmic RBF equalizer, we will proceed to introduce the combined RBF equalization and turbo-coding system and investigate its performance in both fixed QAM and $\mathrm{BbB}$ AQAM schemes.

\section{SYSTEM OVERVIEW}

The structure of the turbo-channel decoder assisted, RBF DFE-aided BbB AQAM scheme is portrayed in Fig. 2. The output of the Jacobian RBF DFE provides the a posteriori log-likelihood ratio (LLR) of the transmitted bits based on the a posteriori likelihood values of each legitimate $\mathcal{M}$-QAM 


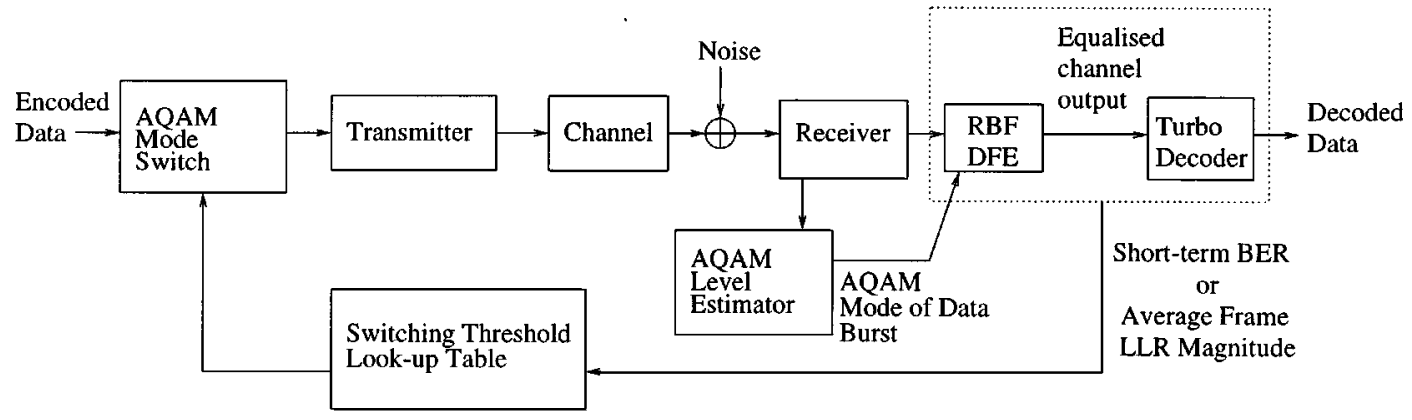

Fig. 2. System schematic of the joint adaptive modulation and RBF equalizer scheme using turbo coding.

symbol. The LLR of a data bit $u_{k}$ is denoted by $\mathcal{L}\left(u_{k}\right)$, which is defined as the $\log$ of the ratio of the probabilities of the bit being a logical 1 or a logical 0 , which is expressed here as:

$$
\begin{aligned}
\mathcal{L}\left(u_{k}\right) & =\ln \left(\frac{P\left(u_{k}=+1\right)}{P\left(u_{k}=-1\right)}\right) \\
& =L\left(u_{k}=+1\right)-L\left(u_{k}=-1\right) .
\end{aligned}
$$

The LLR of the bits representing the QAM symbols can be obtained from the a posteriori likelihood of the symbol. We provide an example for the 4 QAM mode of our AQAM scheme. The a posteriori likelihood $L_{1}, L_{2}, L_{3}$ and $L_{4}$ of the four possible 4 QAM symbols is given by the Jacobian RBF networks. A 4 QAM symbol is denoted by the bits $U_{0} U_{1}$ and the symbols $\mathcal{J}_{1}, \mathcal{J}_{2}, \mathcal{J}_{3}$, and $\mathcal{J}_{4}$ correspond to $00,01,1011$, respectively. Thus, the a posteriori LLRs of the bits are obtained as follows:

$$
\begin{aligned}
& \mathcal{L}\left(U_{0}\right)=L\left(U_{0}=1\right)-L\left(U_{0}=0\right) \\
& \mathcal{L}\left(U_{1}\right)=L\left(U_{1}=1\right)-L\left(U_{1}=0\right)
\end{aligned}
$$

where

$$
\begin{aligned}
L\left(U_{0}=1\right) & =L\left(U_{0} U_{1}=11 \cup U_{0} U_{1}=10\right) \\
& =\ln \left(e^{P\left(U_{0} U_{1}=11\right) \cdot P\left(U_{0} U_{1}=10\right)}\right)=J\left(L_{4}, L_{3}\right) \\
L\left(U_{0}=0\right) & =L\left(U_{0} U_{1}=01 \cup U_{0} U_{1}=00\right) \\
& =\ln \left(e^{P\left(U_{0} U_{1}=01\right) \cdot P\left(U_{0} U_{1}=00\right)}\right)=J\left(L_{2}, L_{1}\right) \\
L\left(U_{1}=1\right) & =L\left(U_{0} U_{1}=11 \cup U_{0} U_{1}=01\right) \\
& =\ln \left(e^{P\left(U_{0} U_{1}=11\right) \cdot P\left(U_{0} U_{1}=01\right)}\right)=J\left(L_{4}, L_{2}\right) \\
L\left(U_{1}=0\right) & =L\left(U_{0} U_{1}=10 \cup U_{0} U_{1}=00\right) \\
& =\ln \left(e^{P\left(U_{0} U_{1}=10\right) \cdot P\left(U_{0} U_{1}=00\right)}\right) \\
& =J\left(L_{3}, L_{1}\right),
\end{aligned}
$$

and $J\left(\lambda_{1}, \lambda_{2}\right)$ is the Jacobian logarithmic relationship of (4).

Note that the Jacobian RBF equalizer will provide $\log _{2}(\mathcal{M})$ number of bit-LLR values for every $\mathcal{M}$-QAM symbol, which are fed to the turbo-channel decoder as its soft inputs. The turbo decoder iteratively improves the BER of the decoded bits and the detected bits are constituted by the sign of the turbo decoder's soft output. An iterative turbo equalizer can be constructed by feeding back the posteriori information obtained by the turbo decoder to the RBF equalizer. Reference [30] shows approximately 1.5 - and 3-dB channel SNR improvement for fixed-mode BPSK and 4 QAM, respectively, at a BER of $10^{-4}$ after the second turbo-equalizer iteration. The channel used in [30] was a three-path equal-weight Rayleigh fading channel with normalized Doppler frequency of $1.5 \times 10^{-4}$. However, turbo-equalized BbB AQAM constitutes our future research.

The probability of error for the detected bits can be estimated on the basis of the soft output of the turbo decoder. Referring to (9) and assuming $P\left(u_{k}=+1\right)+P\left(u_{k}=-1\right)=1$, the probability of error for the detected bits is given by

$$
P_{\text {error }}\left(u_{k}\right)=\left\{\begin{array}{cc}
1-P\left(u_{k}=+1\right) & \\
=P\left(u_{k}=-1\right), & \text { if } \mathcal{L}\left(u_{k}\right) \geq 0 \\
1-P\left(u_{k}=-1\right) & \\
=P\left(u_{k}=+1\right), & \text { if } \mathcal{L}\left(u_{k}\right)<0 .
\end{array}\right.
$$

The probability of a bit having the value of +1 or -1 can be rewritten in terms of its $\operatorname{LLR} \mathcal{L}\left(u_{k}\right)$ as follows:

$$
\begin{aligned}
& P\left(u_{k}=+1\right)=\frac{1}{1+e^{-\mathcal{L}\left(u_{k}\right)}} \\
& P\left(u_{k}=-1\right)=\frac{1}{1+e^{\mathcal{L}\left(u_{k}\right)}} .
\end{aligned}
$$

Upon substituting (13) into (12), we redefined the probability of error of a detected bit in terms of its LLR as

$$
P_{\text {error }}\left(u_{k}\right)=\frac{1}{1+e^{\left|\mathcal{L}\left(u_{k}\right)\right|}}
$$

where $\left|\mathcal{L}\left(u_{k}\right)\right|$ is the magnitude of $\mathcal{L}\left(u_{k}\right)$. The average short-term probability of bit error within the decoded transmission burst is given by

$$
P_{\text {bit, short-term }}=\frac{\sum_{i=0}^{\mathcal{F}} P_{\text {error }}\left(u_{i}\right)}{\mathcal{F}}
$$

where $\mathcal{F}$ is the number of decoded bits per transmitted burst and $u_{i}$ is the $i$ th decoded bit in the frame. This value, which we will refer to as the estimated short-term BER estimates the actual BER of the frame, where the actual BER is the ratio of the number of bit errors encountered in a data burst to the total number of bits transmitted in that burst.

In the next section we will investigate the performance of various turbo-coding assisted RBF DFE $\mathcal{M}$-QAM schemes based on our simulation results. 


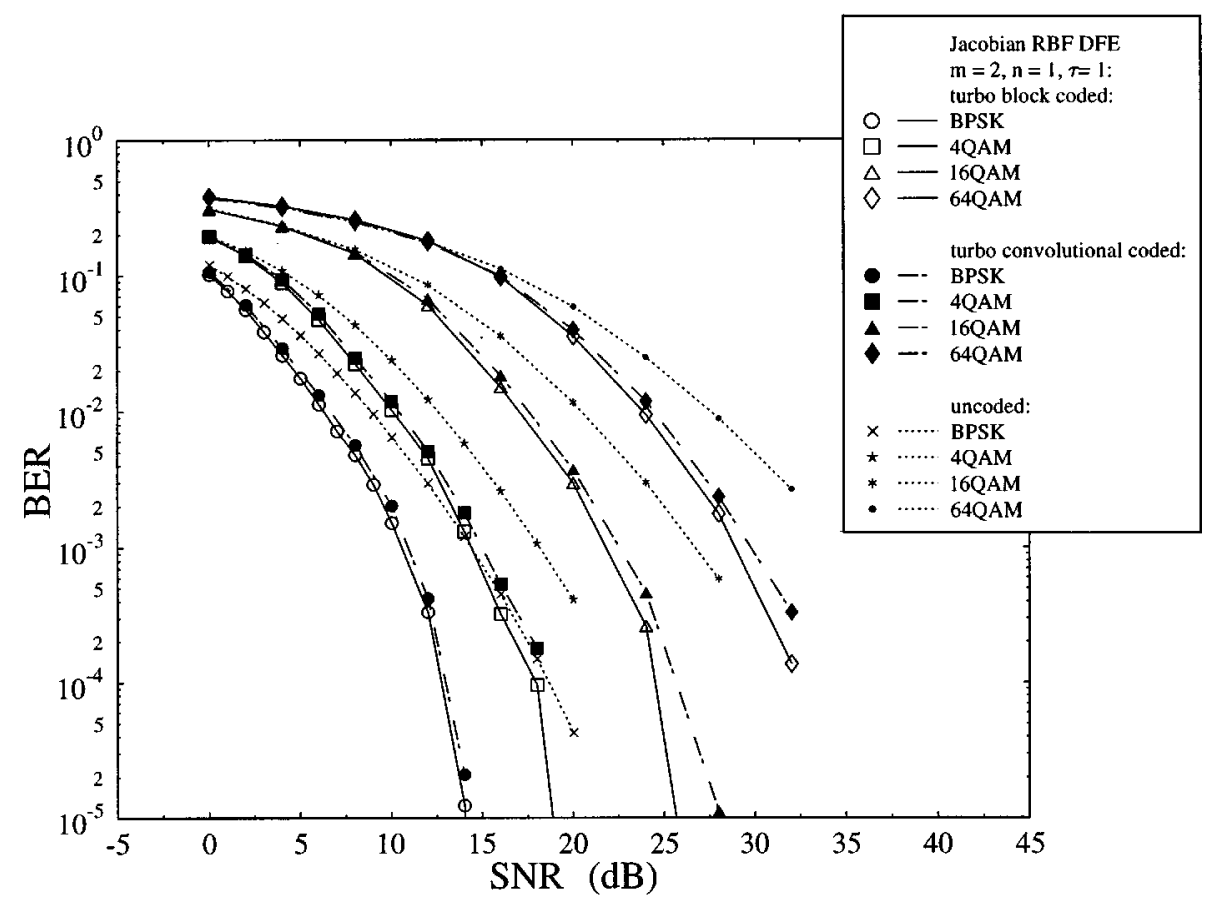

Fig. 3. Probability of bit error versus SNR performance for the Jacobian logarithmic RBF DFE using the turbo codec of Table IV over the dispersive two-path fading channel of Table III for various QAM schemes. The equalizer has a feedforward order of $m=2$, feedback order of $n=1$ and a decision delay of $\tau=1$ symbol. The number of convolutional and BCH turbo decoder iterations is six, while the turbo-interleaver size is fixed to 9984 bits.

\section{TURBo-CODED RBF-EquALIZED $\mathcal{M}$-QAM PERFORMANCE RESULTS}

In this section we characterize the performance of the RBF DFE assisted and turbo-coded $\mathcal{M}$-QAM scheme. High channel code rates in excess of $2 / 3$ are desirable, in order to maximize the BPS throughput of the system. Consequently, block codes were favored as the component code in preference to the Recursive Systematic Convolutional (RSC) code based turbo-coded benchmarker scheme, since turbo-block coding has been shown to perform better for coding rates above $2 / 3$ [31]. This is demonstrated first in Fig. 3, which will be discussed in more depth at a later stage. In our simulations, unless otherwise stated, we hence utilized the turbo-coding parameters given in Table IV 1 and employed the 288- $\mu$ s-duration FMA1 nonspread data transmission burst structure specified in the FRAMES proposal [32]. The turbo encoder used two Bose-Chaudhuri-Hocquenghem $\mathrm{BCH}(31,26)$ block codes in parallel. A 9984-bit random interleaver was used between the two component codes, unless otherwise stated. We used the Log-MAP decoder [27] throughout our investigations, since it offered the same performance as the optimal MAP decoder at a reduced complexity. The RBF DFE used in our simulations had a feedforward order of $m=2$, feedback order of $n=1$ and decision delay of $\tau=1$ symbol. Increasing the feedforward order and decision delay would increase the performance of the fixed mode, especially for higherorder modulation modes, albeit at the expense of an exponential computational complexity increment. For example, according to Table II, the 16 QAM Jacobian RBF DFE having a feedforward order of $m=3$, feedback order of $n=1$ and decision delay

\footnotetext{
${ }^{1}$ The parity bits were not punctured, since block turbo codes suffer from performance loss upon puncturing.
}

TABLE III

Simulation Parameters for Two-Path RAYleigh Fading ChanNel

\begin{tabular}{l|l}
\hline Transmission Frequency & $1.9 \mathrm{GHz}$ \\
Transmission Rate & $2.6 \mathrm{MBd}$ \\
Vehicular Speed & $30 \mathrm{mph}$ \\
Normalised Doppler Frequency & $3.3 \times 10^{-5}$ \\
Channel impulse response (CIR) & $F(z)=0.707+0.707 z^{-1}$ \\
\hline
\end{tabular}

of $\tau=2$ has a computational complexity more than 16 times higher compared to the 16 QAM Jacobian RBF DFE having a feedforward order of $m=2$, feedback order of $n=1$ and decision delay of $\tau=1$. The RBF DFE used correct symbol feedback and we assumed perfect CIR estimation, hence the associated results indicate the system's best-case performance.

We will now investigate the performance of the turbo-coded $\mathcal{M}$-QAM RBF DFE scheme over the wideband Rayleigh fading channel environment of Table III, while the parameters of the turbo codec are given in Table IV. As noted before, Fig. 3 shows the performance of the Jacobian RBF DFE enhanced by both BCH and RSC based turbo coding for various QAM modes. The BCH turbo-coded scheme improves the system's performance by 5, 4, 7, and $8 \mathrm{~dB}$ using BPSK, 4 QAM, 16 QAM and 64 QAM, respectively, at a BER of $10^{-4}$. However, the turbo-coded schemes only start to provide significant BER improvements with respect to the uncoded scheme, once the uncoded BER dips below $10^{-1}$. Our performance comparisons with the turbo-convolutional codec of Table IV given in Fig. 3 demonstrate that the $R=0.72$ turbo-block code provided a marginally better BER performance than the $R=0.75$ RSC 
TABLE IV

TURBo BCH AND RSC CODING PARAMETERS

\begin{tabular}{l||l|l}
\hline & $\mathrm{BCH}$ & $\mathrm{RSC}$ \\
\hline Component code & $\mathrm{BCH}(31,26)$ & $K=5, R=0.5$ \\
Octal generator polynomial & & $G[0]=23_{8} G[1]=35_{8}$ \\
Code rate, $R$ & $0.72=\frac{26}{36} 1$ & 0.75 \\
Turbo interleaver type & Random & Random \\
Turbo interleaver size & 9984 bit & 9984 bit \\
Component decoders & Log-MAP & Log-MAP \\
\hline
\end{tabular}

turbo codec, at the cost of a higher computational complexity. As seen in Table IV, a half rate RSC encoder of constraint length $K=5$ was used in the RSC turbo codec. The generator polynomials expressed in octal terms were set to 23 (for the feedback path) and 35 . Similarly to the turbo $\mathrm{BCH}$ codec, the code rate was set to 0.75 by applying a random puncturing pattern in the RSC encoder. The turbo-interleaver depth was also chosen to be 9984 bits. We employed the same simplified technique as in [33] for estimating the various schemes' relative complexity, where the number of trellis transitions per information bit was the basis of our comparisons. Employing the equations derived in [33], we found that the estimated complexity of $\mathrm{BCH}(31,26)$ turbo codec and $\operatorname{RSC}(2,1,5)$ turbo codec are 2039 ad 1152, respectively. The complexity of the $\mathrm{BCH}(31,26)$ turbo codec is a factor of $(2039 / 1152=) 1.77$ higher than that of the $\operatorname{RSC}(2,1,5)$ turbo codec. Since we are aiming for the best possible system performance, the $\mathrm{BCH}(31,26)$ turbo codec was used instead of the $\operatorname{RSC}(2,1,5)$ turbo codec for our AQAM scheme simulations at the cost of a higher computational complexity.

In our proposed turbo-coding assisted, RBF DFE BbB AQAM scheme of Fig. 2 we invoked the short-term BER estimates of (14) and (15) in controlling the AQAM modem modes. Explicitly, in Fig. 2 the Jacobian RBF DFE LLRs provide an estimate of the BER of the current AQAM burst, and on the basis of this BER estimate the remote AQAM transmitter is instructed by superimposing a 2-bit AQAM mode request message upon the receiver's own transmitted burst in order to invoke the transmission mode deemed to provide the highest BPS throughput, while meeting our target BER requirement [1], [8], [23]. Due to lack of space these issues are beyond the scope of this contribution and we have assumed zero-delay perfect AQAM mode signaling. For a deeper discussion on this topic the reader is referred to [4], while the associated co-channel interference aspects were treated in [34].

\section{Turbo Coding Assisted BbB AQAM RBF DFE PERFORMANCE}

\section{A. System Overview}

As discussed in Section III, the probability of bit errors is related to the magnitude of the bit LLR according to (14). The $\mathrm{BCH}(31,26)$ turbo decoder iteratively improves the BER of the decoded bits. The average value of the LLR magnitude of all bits in the transmission burst after equalization can be used for estimating the average probability of error of the turbo $\mathrm{BCH}(31$, 26) decoded frame. Thus, the average frame LLR magnitude provided by the equalizer can be used as the BbB AQAM mode switching criterion. We define the average frame LLR magnitude as follows:

$$
\mathcal{L}_{\text {average }}=\frac{\sum_{i=0}^{\mathcal{F}}\left|\mathcal{L}\left(u_{i}\right)\right|}{\mathcal{F}}
$$

where $\mathcal{F}$ is the number of data bits per transmitted frame and $u_{i}$ is the $i$ th data bit in the frame.

Again, the schematic of the proposed turbo-coded AQAM RBF DFE arrangement is depicted in Fig. 2. The average frame LLR magnitude of (16) generated by the RBF DFE is compared to a set of switching LLR magnitude thresholds, bearing in mind the current AQAM mode of the equalized data burst. Consequently, a modulation mode is selected for the next transmission burst, based on the current estimated BER upon assuming slowly fading channels. More explicitly, this implies that the similarity of the average frame LLR magnitude of consecutive data bursts can be exploited, in order to set the next modulation mode. The modulation modes utilized in our system are BPSK, 4 QAM, 16 QAM, 64 QAM and no transmission (NO TX). Therefore, the modulation mode is switched according to the average frame LLR magnitude as follows:

$$
\begin{aligned}
& \text { Modulation Mode } \\
& = \begin{cases}\text { NO TX, } & \text { if } \mathcal{L}_{\text {average }} \leq \mathcal{L}_{2}^{\mathcal{M}} \\
\text { BPSK, } & \text { if } \mathcal{L}_{2}^{\mathcal{M}}<\mathcal{L}_{\text {average }} \leq \mathcal{L}_{4}^{\mathcal{M}} \\
4 \mathrm{QAM}, & \text { if } \mathcal{L}_{4}^{\mathcal{M}}<\mathcal{L}_{\text {average }} \leq \mathcal{L}_{16}^{\mathcal{M}} \\
16 \mathrm{QAM}, & \text { if } \mathcal{L}_{16}^{\mathcal{M}}<\mathcal{L}_{\text {average }} \leq P_{64}^{\mathcal{M}} \\
64 \mathrm{QAM}, & \text { if } \mathcal{L}_{64}^{\mathcal{M}}<\mathcal{L}_{\text {average }}\end{cases}
\end{aligned}
$$

where $\mathcal{L}_{i}^{\mathcal{M}}, i=2,4,16,64$ are the switching LLR magnitude thresholds corresponding to the various $\mathcal{M}$-QAM modes. The switching LLR magnitude thresholds corresponding to $\mathcal{M}$-QAM, $\mathcal{L}_{i}^{\mathcal{M}}, i=2,4,16,64$, can be obtained by estimating the average frame LLR magnitude degradation/improvement, upon switching the modulation mode from the current $\mathcal{M}$-QAM mode to a higher/lower number of bits per symbol. The target BER requirement can be met by obtaining the average frame LLR magnitude of each modulation mode corresponding to the estimated channel quality and by activating the specific AQAM mode satisfying this target BER. In adapting the modulation mode, an AQAM mode signaling regime has to be implemented in order to harmonize the operation of the transmitter and receiver, for example using the regime proposed in [35].

\section{B. Simulation Results}

The simulation parameters used in our experiments are listed in Table III, noting that we analyzed the combined AQAM and $\mathrm{RBF}$ DFE scheme in conjunction with $\mathrm{BCH}(31,26)$ turbo coding over the two-path Rayleigh fading channel of Table III. The dicrete-time channel of Table III is a concatenation of 
TABLE V

RANDOM INTERLEAVER SIZES FOR EACH AQAM MODULATION MODE OF (17) TO FACILITATE BbB DECODING AND USING THE 288 MicroseCONDS FMA1 Nonspread Data TRANSMISSION BURST STRUCTURE SPECIFIED IN THE FRAMES PROPOSAL [32]

\begin{tabular}{c||c|c|c|c}
\hline Modulation Mode & BPSK & 4QAM & 16QAM & 64QAM \\
\hline Turbo Interleaver Size & 494 & 988 & 1976 & 2964 \\
\hline
\end{tabular}

the transmitter filter, the wireless channel and the receiver filter. We used square root Nyquist pulse-shaping and we assumed that the pulse-shaping does not introduce intersymbol interference to the overall system. We do acknowledge that a more realistic outdoor mobile channel model may have more than two taps. The symbol-spaced two-path channel had a total dispersion of about $400 \mathrm{~ns}$ at the $2.6 \mathrm{MBd}$ signaling rate used, which is associated with a path-length difference of 128 $\mathrm{m}$ prevalent in pico- or microcellular scenarios. The wideband fading channel was burst-invariant, implying that during a transmission burst the CIR was considered time-invariant. In our simulations, we used the Jacobian RBF DFE having a feedforward order of $m=2$, feedback order of $n=1$ and decision delay of $\tau=1$. We used the $\operatorname{BCH}(31,26)$ code of Table IV as the turbo-component code and a variable-length random interleaver depending on the BPS throughput of the modulation mode used, as given in Table $\mathrm{V}$, in order to enable BbB decoding. We also note that we do not utilize a channel interleaver because of the less bursty nature of the error distribution in conjunction with the AQAM scheme, than in a fixed high-order modulation schemes where dropping the number of bits per symbol is infeasible. The AQAM scheme always attempts to invoke the appropriate modulation mode for reducing the probability of encountering a received transmitted burst having a high instantaneous BER. The modulation modes utilized in our system are BPSK, 4 QAM, 16 QAM, 64 QAM and NO TX. During the beginning of a sequence of bursts, where the channel conditions are not known precisely, BPSK namely, the most robust modulation mode, is used. We also assume that the receiver has perfect knowledge of the modulation mode used in its received transmission burst. In a practical system, the transmitter notifies the receiver of all the AQAM mode changes and control symbols must be used for conveying the modulation mode employed by the transmitter to the receiver, for example using the regime of [35].

In our experiments, we obtained the LLR magnitude degradation/improvement upon switching from each AQAM mode to all other legitimate modes under the same instantaneous channel conditions. As an example, Fig. 4 shows the estimated short-term BER - defined in (15) - that would be encountered upon switching to all possible AQAM modes after $\mathrm{BCH}(31,26)$ turbo decoding versus the average frame LLR magnitude of 4QAM before decoding, which was the current AQAM mode. Each point in Fig. 4 represents the decoder's estimated short-term BER (specified by the $y$-axis) for a particular received data burst using the corresponding modulation mode and the average frame LLR magnitude (specified by the $x$-axis) of that burst before decoding, when the current AQAM

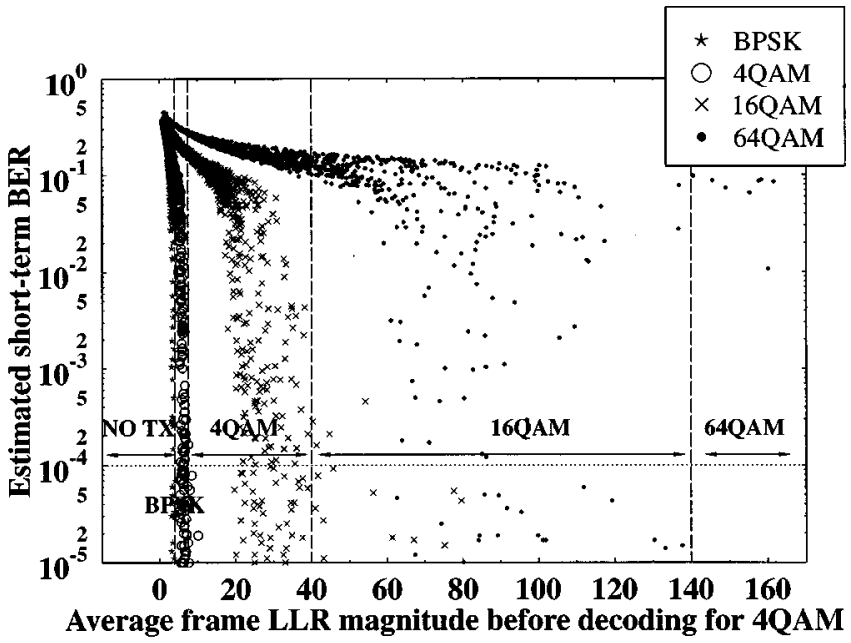

Fig. 4. The estimated short-term BER for all the possible $\mathrm{BCH}(31,26)$ turbodecoded AQAM modes versus the average frame LLR magnitude of 4QAM over the two-path Rayleigh fading channel of Table III. The figure illustrates the expected spread of the short-term BER of all turbo-decoded modem modes given a certain average frame LLR magnitude value in conjunction with 4QAM as the current modem mode.

mode is 4 QAM, which was calculated using (16). In order to maintain the target BER of $10^{-4}$, Fig. 4 demonstrates how each switching LLR magnitude $\mathcal{L}_{i}^{4}, i=2,4,16,64$ is obtained after averaging the LLR occurrences seen in the figure. More explicitly, the average frame LLR magnitudes encountered in the 4 QAM transmission burst would have to be 4.0, 7.5, 40.0 and 100.0, before switching to BPSK, 4 QAM, 16 QAM and 64 QAM AQAM bursts under the same channel conditions, leading to an estimated BER of $10^{-4}$ after $\mathrm{BCH}(31,26)$ turbo decoding, as seen in bold in Table VI. For example, if the average LLR magnitude of the received 4 QAM transmission burst, $\mathcal{L}_{\text {average }}$ is in the range of $100>\mathcal{L}_{\text {average }} \geq 40$, in Fig. 4 the modulation mode is switched from 4 QAM to 16 QAM for the next AQAM burst, since the BER of this 16 QAM transmission burst is estimated to be below the target BER of $10^{-4}$. Note that due to the spreading of the average frame LLR magnitude versus the short-term BER curve in Fig. 4-especially for higher-order AQAM modes-the threshold is estimated from the mean of this dynamic range. Using the same method for the other modulation modes, the switching LLR magnitude thresholds were obtained for the turbo-decoded target BER of $10^{-4}$, as listed in Table VI.

Fig. 5 shows the performance comparison of the AQAM Jacobian RBF DFE scheme in conjunction with turbo $\mathrm{BCH}(31,26)$ coding for a target BER of $10^{-4}$ along with its constituent turbocoded fixed QAM modes. Fig. 5 also shows the BER and BPS performance of the AQAM/RBF DFE scheme without turbo coding, with the short-term BER as the switching criterion as described in [23] for performance comparison. The switching BER thresholds of the AQAM/RBF DFE scheme without turbo coding were listed in Table VII.

Referring to Fig. 5, the turbo $\mathrm{BCH}(31,26)$-coded BPS performance was better than that of the uncoded scheme for the average channel SNR range of 0 to $26 \mathrm{~dB}$ with a maximum SNR 
TABLE VI

THE Switching LLR MAgnitude Thresholds $\mathcal{L}^{\mathcal{M}}$ OF THE Turbo BCH(31,26)-Assisted RBF DFE BbB AQAM SCHEME FOR THE TARGET BER OF $10^{-4}$

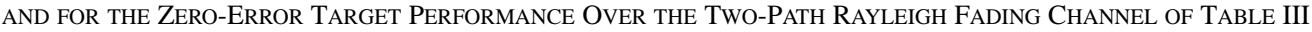

\begin{tabular}{c||c|c|c|c||c|c|c|c||c|c|c|c}
\hline \multicolumn{1}{l||}{} & \multicolumn{4}{c||}{ RBF DFE with correct decision feedback } & \multicolumn{4}{c|}{ with detected decision feedback } \\
\hline \multicolumn{1}{l||}{} & \multicolumn{3}{c|}{ target BER of $10^{-4}$} & \multicolumn{3}{c|}{ target zerro-error } & \multicolumn{4}{c|}{ target BER of $10^{-4}$} \\
\hline & $\mathcal{L}_{2}^{\mathcal{M}}$ & $\mathcal{L}_{4}^{\mathcal{M}}$ & $\mathcal{L}_{\mathbf{1 6}}^{\mathcal{M}}$ & $\mathcal{L}_{64}^{\mathcal{M}}$ & $\mathcal{L}_{2}^{\mathcal{M}}$ & $\mathcal{L}_{4}^{\mathcal{M}}$ & $\mathcal{L}_{16}^{\mathcal{M}}$ & $\mathcal{L}_{64}^{\mathcal{M}}$ & $\mathcal{L}_{2}^{\mathcal{M}}$ & $\mathcal{L}_{4}^{\mathcal{M}}$ & $\mathcal{L}_{16}^{\mathcal{M}}$ & $\mathcal{L}_{64}^{\mathcal{M}}$ \\
\hline NO TX & 8.0 & $\mathbf{1 7 . 0}$ & 90.0 & 380.0 & 10.0 & 30.0 & 280.0 & 1000.0 & 10.0 & 35.0 & 2500.0 & 10000.0 \\
\hline BPSK & 8.0 & $\mathbf{1 7 . 0}$ & 90.0 & 380.0 & 10.0 & 30.0 & 280.0 & 1000.0 & 10.0 & 35.0 & 2500.0 & 10000.0 \\
\hline 4QAM & $\mathbf{4 . 0}$ & $\mathbf{7 . 5}$ & $\mathbf{4 0 . 0}$ & $\mathbf{1 4 0 . 0}$ & 8.0 & 12.0 & 100.0 & 350.0 & 4.0 & 22.0 & 250.0 & 1000.0 \\
\hline 16QAM & 2.0 & 3.0 & 11.5 & 55.0 & 3.0 & 5.0 & 30.0 & 120.0 & 2.0 & 4.5 & 90.0 & 1000.0 \\
\hline 64QAM & 1.7 & 2.2 & 6.2 & 30.0 & 2.5 & 3.0 & 13.0 & 70.0 & 1.0 & 3.0 & 50.0 & 800.0 \\
\hline
\end{tabular}

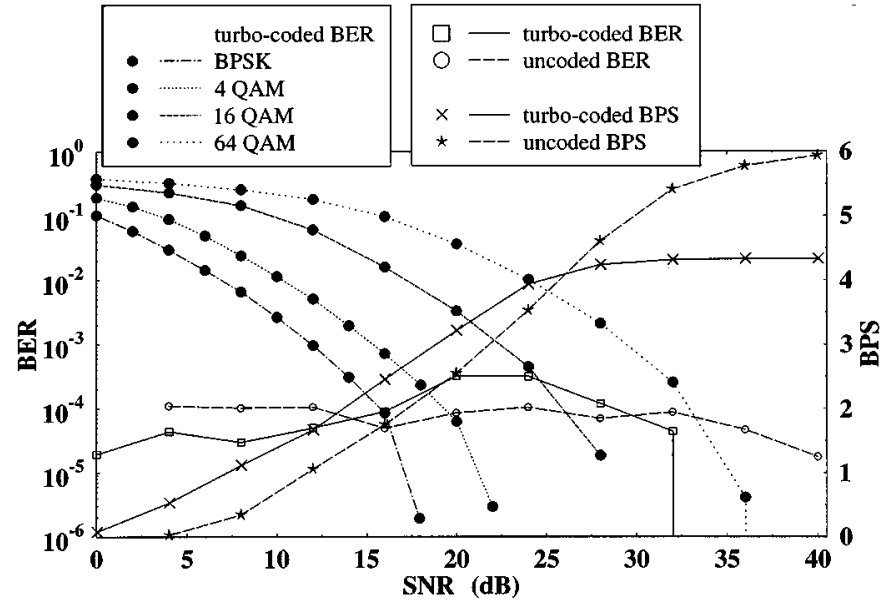

Fig. 5. BER and BPS performance of the turbo BCH(31,26)-coded AQAM Jacobian RBF DFE for a data-transmission target BER of $\mathbf{1 0}^{-4}$ using the parameters listed in Table III. The modem mode used the "before-decoding" LLR switching criterion for this scheme with the thresholds listed in Table VI. The Jacobian RBF DFE had a feedforward order of $m=2$, feedback order of $n=1$ and decision delay of $\tau=1$ symbol. The turbo-coding parameters were given in Table IV and the number of turbo-decoder iterations was six. The turbo-interleaver size was fixed according to the modulation modes used, as shown in Table V.

TABLE VII

SWITCHING BER THRESHOLDS $P_{i}^{\mathcal{M}}$ OF THE UNCODED RBF DFE BbB AQAM SCHEME FOR THE TARGET BER OF $10^{-4}$ OVER THE TWO-PATH RAYLEIGH FADING CHANNEL OF TABLE III

\begin{tabular}{c|c|c|c|c}
\hline & $P_{2}^{\mathcal{M}}$ & $P_{4}^{\mathcal{M}}$ & $P_{16}^{\mathcal{M}}$ & $P_{64}^{\mathcal{M}}$ \\
\hline NO TX & $9 \times 10^{-5}$ & $1 \times 10^{-15}$ & 0.0 & 0.0 \\
\hline BPSK & $1 \times 10^{-4}$ & $1 \times 10^{-15}$ & 0.0 & 0.0 \\
\hline 4QAM & $1.5 \times 10^{-2}$ & $1 \times 10^{-4}$ & $1 \times 10^{-45}$ & 0.0 \\
\hline 16QAM & $1.2 \times 10^{-1}$ & $5 \times 10^{-2}$ & $1 \times 10^{-4}$ & $1 \times 10^{-50}$ \\
\hline $64 \mathrm{QAM}$ & $2.2 \times 10^{-1}$ & $1.5 \times 10^{-1}$ & $3 \times 10^{-2}$ & $1 \times 10^{-4}$ \\
\hline
\end{tabular}

gain of $4 \mathrm{~dB}$ at a channel SNR of $0 \mathrm{~dB}$. However, at high SNRs the BPS performance is limited by the turbo-coding rate of the system to a maximum BPS throughput of $26 / 36 \cdot 6=4.33$. The turbo $\mathrm{BCH}(31,26)$-coded AQAM system also exhibited a superior BER performance, when compared to the uncoded system for the average channel SNR range of 0 to $16 \mathrm{~dB}$ and for the range above $28 \mathrm{~dB}$. The turbo $\mathrm{BCH}(31,26)$-coded AQAM system failed to achieve the target BER of $10^{-4}$ in the SNR range of $16 \mathrm{~dB}$ to $28 \mathrm{~dB}$. This is because the spread nature of the short-term BER versus LLR magnitude curves in Fig. 4 leads to inaccuracies in obtaining these LLR magnitude thresholds, especially for $\mathcal{L}_{16}^{\mathcal{M}}$ and $\mathcal{L}_{64}^{\mathcal{M}}$. These inaccuracies affect the switching performance for the SNR range of $16 \mathrm{~dB}$ to $28 \mathrm{~dB}$. The spread nature of the short-term BER versus LLR magnitude curves in Fig. 4 is due to a number of factors and these investigations are set aside for future work.

Since the estimated short-term BER in Fig. 4 is a somewhat erratic function of the turbo-decoder's input LLR, the switching LLR values have to be conservative, if the target BER cannot be exceeded. For the BER $=10^{-4}$ scenario the switching LLR can be adjusted to be near the upper end of the LLR-range observed in Fig. 4. When aiming for virtually error-free communications, an even more conservative LLR threshold has to be chosen, in order not to precipitate a plethora of transmission errors, even at the cost of thereby reducing the achievable BPS throughput of the system. Recent studies of the SNR-dependent AQAM switching thresholds can be found in [35]-[37]. Our future research will explore the feasibility of finding a real-time adaptive learning algorithm for providing optimized switching thresholds for the AQAM scheme online. Fig. 6 shows the BER and BPS performance of the near-zero target BER turbo-coded AQAM Jacobian RBF DFE scheme with the more conservative, i.e., increased LLR magnitude based switching thresholds listed in Table VI. The BER and BPS performance of the uncoded AQAM RBF DFE system is also characterized in the figure for comparison. The BPS performance of the coded system was better, than that of the uncoded AQAM system for the average channel SNR range of $0-15 \mathrm{~dB}$, as evidenced by Fig. 6. However, the BPS throughput was limited by the $26 / 36$ coding rate of the system to a maximum value of $6 \cdot 26 / 36=4.33$ at high channel SNRs. This suggests that the best overall BER/BPS performance is achieved by our system, if we add the AQAM option of switching off the turbo $\mathrm{BCH}(31,26)$ code under high SNR conditions, namely around $25 \mathrm{~dB}$. This allows us to attain a BPS of 6 in this SNR region, as characterized by the uncoded BPS curve. Note that the high SNR required for the high-order 


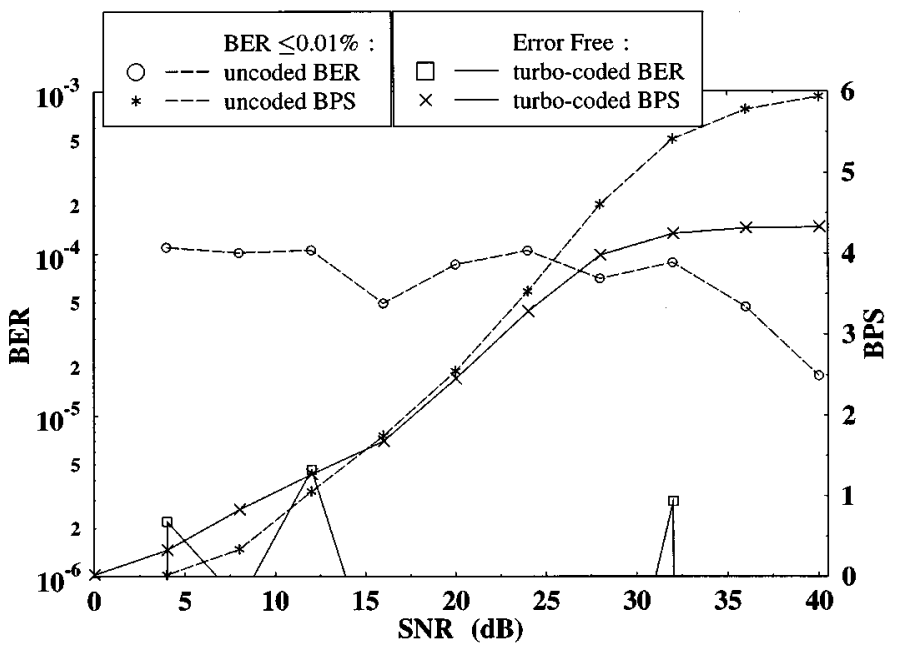

Fig. 6. The BER and BPS performance of the joint AQAM/Jacobian RBF DFE with turbo $\mathrm{BCH}(31,26)$ coding for near-zero target BER transmission using the parameters listed in Table III. The modem mode used "before decoding" LLR switching criterion for this scheme with the thresholds listed in Table VI. The Jacobian RBF DFE had a feedforward order of $m=2$, feedback order of $n=1$ and decision delay of $\tau=1$ symbol. The turbo-coding parameter is given in Table IV and the number of turbo-decoder iterations is 6 . The turbo-interleaver size is fixed according to the modulation mode used as shown in Table V.

AQAM modes does not lead to high intercell interference, unlike schemes that use power control in an effort to maintain a given SNR and thereby spilling energy into adjacent cells. In other words, the AQAM modem unobtrusively adjust its own modulation mode in order to best exploit the SNR available and to attain the highest possible BPS throughput in arbitrary unknown propagation environments.

We will now investigate the effects of imperfect channel estimation and error propagation of the RBF DFE on our system. We will first investigate the effects of error propagation. Again, the simulation parameters used are listed in Table III and the turbo-interleaver length varies according to the modulation mode used, as seen in Table V. Fig. 7 shows the performance degradation due to error propagation in the RBF DFEs decision feedback loop. We first study the performance using perfect channel estimation. The performance degradation increases when using high-order QAM modes. The performance degradations at a BER of $10^{-4}$ are $1,2,8$, and $12 \mathrm{~dB}$ for BPSK, 4 QAM, 16 QAM, and 64 QAM, respectively. Hence a new set of AQAM switching LLRs were obtained according to Fig. 4, but with the detected decision feedback. The corresponding values are given at the right half of Table VI. In conclusion of these investigations Fig. 8 shows that the BER performance of the turbo-coded AQAM/RBF DFE, which is similar with and without the effects of error propagation. This shows that the AQAM switching mechanism is capable of maintaining the targeted BER, since the RBF DFE retains its ability to estimate the channel quality even in the presence of decoding errors. The BPS performance degrades however significantly, especially at medium SNRs due to the more grave performance degradation of the higher-order AQAM modes induced by the error propagation of the RBF DFE. For example, the BPS throughput reduction is approximately 1.6 BPS at a channel

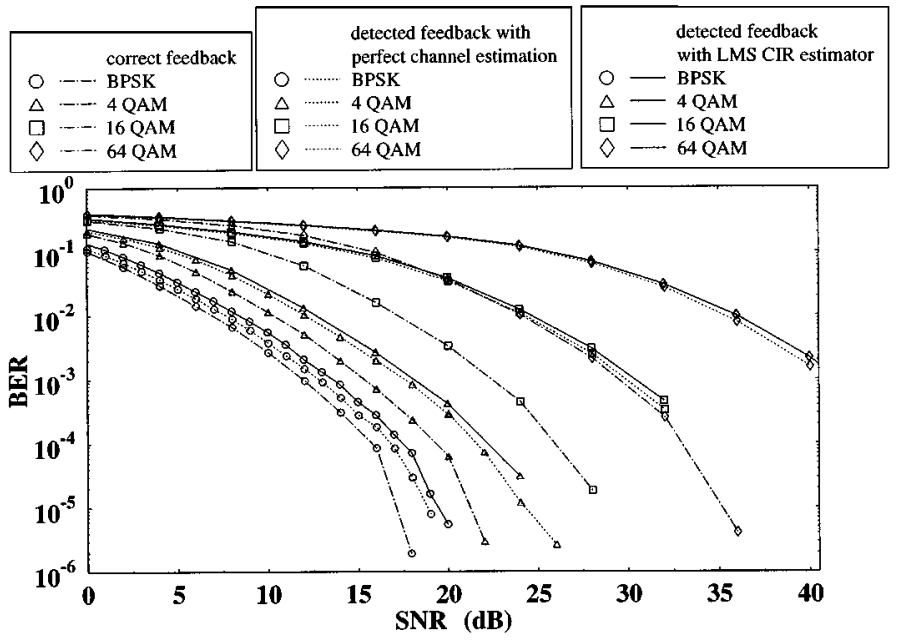

Fig. 7. Probability of bit error versus SNR performance comparison for the Jacobian logarithmic RBF DFE using the turbo codec of Table IV with error propagation and without error propagation over the dispersive two-path fading channel of Table III for various QAM schemes. The LMS channel estimator has a step-size of 0.1 . The equalizer has a feedforward order of $m=2$, feedback order of $n=1$ and a decision delay of $\tau=1$ symbol. The number of convolutional and $\mathrm{BCH}$ turbo-decoder iterations is six, while the turbo-interleaver size is fixed according to the modulation mode used as shown in Table V.

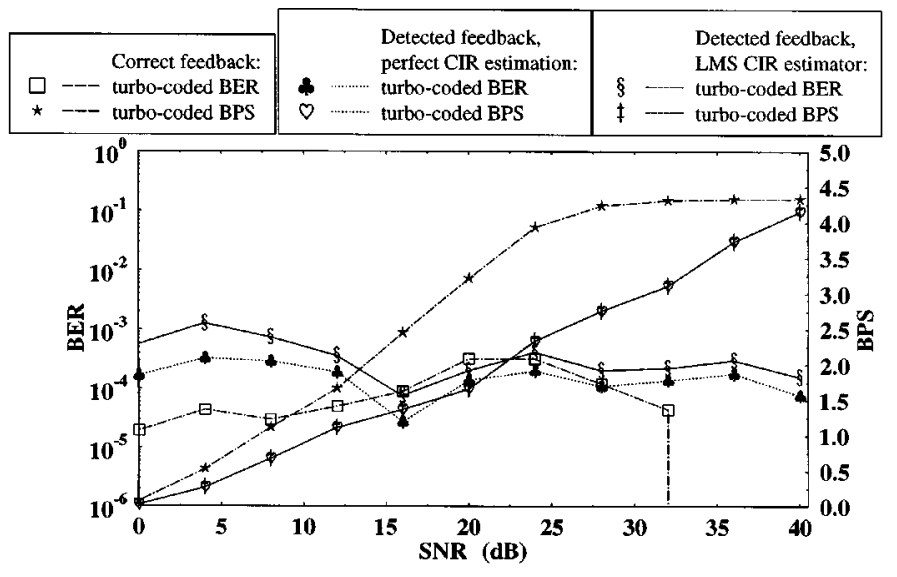

Fig. 8. The BER and BPS performance of the turbo $\mathrm{BCH}(31,26)$-coded joint AQAM/Jacobian RBF DFE with correct and detected decision feedback for a data-transmission target BER of $\mathbf{1 0}^{-4}$ using the parameters listed in Table III. The LMS channel estimator has a step-size of 0.1. The modem mode used "before decoding" LLR switching criterion for this scheme with the thresholds listed in Table VI for correct decision feedback and detected decision feedback. The Jacobian RBF DFE had a feedforward order of $m=2$, feedback order of $n=1$ and decision delay of $\tau=1$ symbol. The turbo-coding parameter is given in Table IV and the number of turbo-decoder iterations is 6 . The turbo-interleaver size is fixed according to the modulation mode used as shown in Table V. Observe that the BPS legends associated with perfect as well as LMS-based CIR estimation, where in both cased using detected feedback, coincide with each other.

SNR of $24 \mathrm{~dB}$. Further research is required for mitigating the effects of error propagation, especially in high-order AQAM modes, in order to improve the BER performance.

We will now investigate the effects of imperfect channel estimation on the performance of our system. Returning briefly to Fig. 7 we observe that there is only marginal performance degradation for the individual fixed QAM modes, when we estimate the CIR using an LMS channel estimator [38] having a step-size of 0.1. This is also corroborated in Fig. 8 in the context 
of AQAM. The average BER reaches $10^{-3}$, which is an order of magnitude higher than the target BER of $10^{-4}$. Nevertheless, in order to achieve the target BER of $10^{-4}$, we have to adjust the LLR magnitude based switching thresholds to more conservative values for compensating for the imperfect CIR estimation, as seen in Table VI. Our experimental results not included here show that this is achieved at a negligible reduction of the throughput.

Overall, we have demonstrated that an RBF assisted BbB AQAM scheme is capable of adapting to the time-variant channel quality fluctuations, estimated for example by the RBF equalizer of the system, in order to achieve a given target BER, while maintaining the highest possible BPS throughput.

\section{CONCLUSION}

In conclusion, the performance of the RBF DFE has been documented in the context of fixed-mode modulation schemes in [6] and [7]. However, there is little information concerning its potential in either BbB AQAM or in FEC-coded scenarios, despite the advantageous interactions of RBF-aided DFE BbB AQAM in conjunction with turbo FEC, which were demonstrated in our preliminary work [8]. In this contribution the complexity of the RBF DFE was reduced upon invoking the Jacobian logarithmic approximation of (4) for avoiding the evaluation of exponential functions in the RBF DFE. We have shown that the average frame LLR at the input of the turbo decoder can be used as an effective metric for adapting the AQAM modes according to the time-variant channel conditions.

The turbo-coded AQAM RBF DFE system exhibited a better BPS performance, when compared to the uncoded system at low to medium channel SNRs, as evidenced by Fig. 5. The same figure also showed an improved coded BER performance at higher channel SNRs. A virtually error-free turbo-coded AQAM scheme was also characterized in Fig. 6. Our future work is targeted at invoking turbo equalization and adaptive beam-steering, in order to reduce the effects of co-channel interference.

\section{REFERENCES}

[1] W. T. Webb and R. Steele, "Variable rate QAM for mobile radio," IEEE Trans. Commun., vol. 43, pp. 2223-2230, July 1995.

[2] S. Sampei, S. Komaki, and N. Morinaga, "Adaptive modulation/TDMA scheme for large capacity personal multimedia communications systems," IEICE Trans. Commun., vol. E77-B, pp. 1096-1103, Sept. 1994.

[3] A. J. Goldsmith and S. G. Chua, "Variable rate variable power MQAM for fading channels," IEEE Trans. Commun., vol. 45, pp. 1218-1230, Oct. 1997.

[4] J. M. Torrance and L. Hanzo, "Latency and networking aspects of adaptive modems over slow indoors Rayleigh fading channel," IEEE Trans. Veh. Technol., vol. 48, pp. 1237-1251, July 1999.

[5] C. H. Wong and L. Hanzo, "Channel capacity upperbound of a wideband burst-by-burst adaptive modem," in Proc. IEEE Veh. Technol. Conf., May 1999, pp. 1851-1855.

[6] S. Chen, S. McLaughlin, and B. Mulgrew, "Complex-valued radial basis function network, Part II: Application to digital communications channel equalization," EURASIP Signal Processing, vol. 36, pp. 175-188, Mar. 1994.

[7] M. S. Yee and L. Hanzo, "Multi-level radial basis function network based equalisers for Rayleigh channels," in Proc. IEEE Veh. Technol. Conf., May 16-19, 1999, pp. 707-711.

[8] M. S. Yee, T. H. Liew, and L. Hanzo, "Block turbo coded burst-byburst adaptive radial basis function decision feedback equaliser assisted modems," in Proc. IEEE Veh. Technol. Conf., vol. 3, Amsterdam, The Netherlands, Sept. 1999, pp. 1600-1604.
[9] G. J. Gibson, S. Siu, and C. F. N. Cowan, "The application of nonlinear structures to the reconstruction of binary signals," IEEE Trans. Signal Processing, vol. 39, pp. 1877-1884, Aug. 1991.

[10] —, "Multi-layer perceptron structures applied to adaptive equalizers for data communications," in ICASSP, IEEE Int. Conf. Acoust., Speech, Signal Processing, May 1989, vol. 2, pp. 1183-1186.

[11] S. Siu, G. J. Gibson, and C. F. N. Cowan, "Decision feedback equalization using neural network structures and performance comparison with standard architecture," IEE Proc., vol. 137, pp. 221-225, Aug. 1990.

[12] S. Chen, G. J. Gibson, and C. F. N. Cowan, "Adaptive channel equalization using a polynomial-perceptron structure," Proc. Inst. Elect. Eng., vol. 137, pp. 257-264, Oct. 1990.

[13] W. S. Gan, J. J. Soraghan, and T. S. Durrani, "New functional-link based equaliser," Electron. Lett., vol. 28, pp. 1643-1645, Aug. 1992.

[14] T. Kohonen, O. Simula, A. Visa, and J. Kangas, "Engineering applications of the self-organizing map," Proc. IEEE, vol. 84, pp. 1358-1384, Oct. 1996.

[15] J. C. Sueiro, A. A. Rodriguez, and A. R. F. Vidal, "Recurrent radial basis function networks for optimal symbol-by-symbol equalization," EURASIP Signal Processing, vol. 40, pp. 53-63, Oct. 1994.

[16] S. Chen, B. Mulgrew, and P. M. Grant, "A clustering technique for digital communications channel equalization using radial basis function networks," IEEE Trans. Neural Networks, vol. 4, pp. 570-579, July 1993

[17] S. Chen, B. Mulgrew, and S. McLaughlin, "Adaptive Bayesian equalizer with decision feedback," IEEE Trans. Signal Processing, vol. 41, pp. 2918-2927, Sept. 1993.

[18] H. L. V. Trees, Detection, Estimation and Modulation Theory, Part 1. New York: Wiley, 1968.

[19] S. Chen, S. McLaughlin, B. Mulgrew, and P. M. Grant, "Bayesian decision feedback equaliser for overcoming co-channel interference," Proc. Inst. Elect. Eng., vol. 143, pp. 219-225, Aug. 1996.

[20] S. Chen, B. Mulgrew, E.-S. Chng, and G. J. Gibson, "Space translation properties and the minimum-BER linear-combiner DFE," Proc. Inst. Elect. Eng., vol. 145, pp. 316-322, Oct. 1998.

[21] L. Hanzo, W. T. Webb, and T. Keller, Single-and Multicarrier Quadrature Amplitude Modulation. New York: Wiley, IEEE, 2000.

[22] M. S. Yee and L. Hanzo, "Upper bound performance of radial basis function decision feedback equalised burst-by-burst adaptive modulation," in ECMCS'99 Krakow, Poland, June 24-26, 1999, CD-ROM.

[23] M. S. Yee and L. Hanzo, "Radial basis function decision feedback equaliser assisted burst-by-burst adaptive modulation," in Proc. IEEE Globecom'99, Rio de Janeiro, Brazil, Dec. 5-9, 1999, pp. 2183-2187.

[24] C. Berrou, A. Glavieux, and P. Thitimajshima, "Near Shannon limit error-correcting coding and decoding: Turbo codes," in Proc. IEEE Int. Conf. Commun.: IEEE, May 1993, pp. 1064-1070.

[25] P. Robertson, P. Hoeher, and E. Villebrun, "Optimal and sub-optimal maximum a posteriori algorithms suitable for turbo decoding," Euro. Trans. Telecommun., vol. 8, 1997.

[26] D. S. Broomhead and D. Lowe, "Multivariable functional interpolation and adaptive networks," Complex Systems, vol. 2, pp. 321-355, 1988.

[27] P. Robertson, E. Villebrun, and P. Hoeher, "A comparison of optimal and sub-optimal MAP decoding algorithms operation in the log domain," in IEEE Int. Conf. Commun., June 1995, vol. 2, pp. 1009-1013.

[28] J. Erfanian, S. Pasupathy, and G. Gulak, "Reduced complexity symbol detectors with parallel structures for ISI channels," IEEE Trans. Commun., vol. 42, pp. 1661-1671, Feb./Mar./Apr. 1994.

[29] S. K. Patra and B. Mulgrew, "Computational aspects of adaptive radial basis function equalizer design," in IEEE Int. Symp. Circuits Syst. ISCAS'97, June 1997, vol. 1, pp. 521-524

[30] M. S. Yee, B. L. Yeap, and L. Hanzo, "Radial basis function assisted turbo equalization," in Proc. IEEE Veh. Technol. Conf., May 15-18, 2000.

[31] J. Hagenauer, E. Offer, and L. Papke, "Iterative decoding of binary block and convolutional codes," IEEE Trans. Inform. Theory, vol. 42, pp. 429-445, Mar. 1996.

[32] A. Klein, R. Pirhonen, J. Sköld, and R. Suoranta, "FRAMES multiple access mode 1-Wideband TDMA with and without spreading," in Proc. PIMRC'97, Sept. 1997, pp. 37-41.

[33] T. Liew, J. Pliquett, B. Yeap, L.-L. Yang, and L. Hanzo, "Concatenated space time block codes and TCM, turbo TCM, convolutional as well as turbo codes," in GLOBECOM 2000, San Francisco, CA, Nov. 27-Dec. 1, 2000, pp. 2292-2301.

[34] J. M. Torrance, L. Hanzo, and J. Keller, "Interference aspects of adaptive modems over slow Rayleigh fading channel," IEEE Trans. Veh. Technol., vol. 48, pp. 1527-1545, Sept. 1999.

[35] J. M. Torrance and L. Hanzo, "Optimization of switching levels for adaptive modulation in a slow Rayleigh fading channel," Electron. Lett., vol. 32, pp. 1167-1169, June 1996 
[36] B. Choi, M. Münster, L. Yang, and L. Hanzo, "Performance of rake receiver assisted adaptive-modulation based cdma over frequency selective slow Rayleigh fading channels," Electron. Lett., vol. 37, pp. 247-248, Feb. 15, 2001

[37] J. Torrance and L. Hanzo, "Demodulation level selection in adaptive modulation," Electron. Lett., vol. 32, pp. 1751-1752, Sept. 12, 1996.

[38] J. G. Proakis, Digital Communications. New York: McGraw-Hill, 1995.

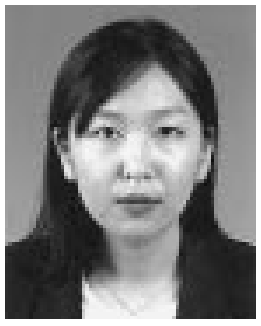

M. S. Yee (S'97-A'00) received the B.Eng. degree in electronics engineering and the Ph.D. degree in telecommunications in 2001, both from the University of Southampton, U.K.

Currently, she is continuing her research as a Postdoctoral Research Fellow. Her research interests are in the field of neural network-based algorithms and their application to various wireless communications problems. She has published numerous papers in this field

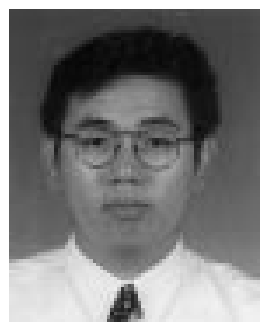

T. H. Liew received the B.Eng. degree in electronics engineering and the Ph.D. degree in telecommunications in 2001, both from the University of Southampton, U.K.

Currently, he is continuing his research as a Postdoctoral research fellow. His research interests are associated with coding and modulation for wireless channels, space-time coding, adaptive transceivers, etc. He has published his research results widely.

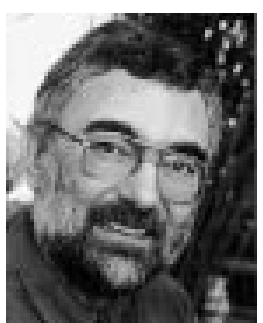

Lajos Hanzo (M'91-SM'92) graduated in electronics in 1976. He received the Ph.D. degree from the Technical University of Budapest, Hungary, in 1983.

During his 25-year career in telecommunications, he has held various research and academic posts in Hungary, Germany, and the U.K. Since 1986, he has been with the Department of Electronics and Computer Science, University of Southampton, U.K. and has been a consultant with Multiple Access Communications Ltd., U.K. Currently, he holds the chair in Telecommunications. He coauthored five books on mobile radio communications, has published more than 300 research papers, organized and chaired conference sessions, presented overview lectures, and was awarded a number of distinctions. He is currently managing a research team that is working on a range of research projects in the field of wireless multimedia communications under the auspices of the Engineering and Physical Sciences Research Council (EPSRC) U.K., the European IST Programme, and the Mobile Virtual Centre of Excellence (VCE). He also provides a range industrial training courses. 